\title{
Siglenverzeichnis und Abkürzungen
}

\section{Werke Kants}

Die Schriften Kants werden zitiert nach der Band- und ggf. auch der Seitenzahl der Akademie Ausgabe von Kants Gesammelten Schriften, Berlin 1900 ff. Die Zitate der Kritik der reinen Vernunft erfolgen ebenfalls nach der Akademie-Ausgabe [im Folgenden: KrV mit der Paginierung der Erstauflage (A) bzw. Zweitauflage (B)].

(AA) Kants Gesammelte Schriften [„Akademieausgabe“], Königlich Preußische Akademie der Wissenschaften, Berlin $1900 \mathrm{ff}$.

(KrV) Kritik der reinen Vernunft (1781/87)

(Prol.) Prolegomena zu einer jeden künftigen Metaphysik, die als Wissenschaft wird auftreten können, eingel. u. mit Anm. hg.v. Konstantin Pollok, Hamburg 2001.

\section{Werke Jacobis}

Jacobi, Friedrich H. ([1815] 2004): „Einleitung in des Verfassers sämmtliche philosophische Schriften“, in: Schriften zum transzendentalen Idealismus. Werke, Bd. 2,1, hg.v. Walter Jaeschke und Irmgard-Maria Piske unter Mitarbeit von Catia Goretzki, Hamburg, S. 375-433. [Jacobi: Einleitung, JWA 2,1]

Jacobi, Friedrich H. ([1787] 2004): „David Hume über den Glauben oder Idealismus und Realismus. Ein Gespräch“, in: Schriften zum transzendentalen Idealismus. Werke, Bd. 2,1, hg.v. Walter Jaeschke und Irmgard-Maria Piske unter Mitarbeit von Catia Goretzki, Hamburg, S. 5-112. [Jacobi: David Hume, JWA 2,1]

Jacobi, Friedrich Heinrich ([1785] 2000): Über die Lehre des Spinoza in Briefen an den Herrn Moses Mendelssohn, hg.v. Marion Lauschke, Hamburg. [Jacobi: Über die Lehre des Spinoza]

(JWA) Jacobi, Friedrich H.: Werke. Gesamtausgabe, hg. von Klaus Hammacher, Walter Jaeschke, Hamburg/Stuttgart-Bad Cannstatt $1998 \mathrm{ff}$. 


\section{Werke Fichtes}

Der vorliegenden Arbeit liegt der Text A zugrunde, d. h. die Grundlage der gesammten Wissenschaftslehre als Handschrift für seine Zuhörer, welche Fichte bogenweise an die Hörer seiner Privatvorlesung in Jena als Werk im Zeitraum vom 14. Juni 1794 bis August 1775 ausgegeben hat und die 1794/95 bei Christian Ernst Gabler in Leipzig verlegt wurde. Die Varianten B („Neue unveränderte Auflage“ bei Johann Friedrich Cotta 1802 in Tübingen) und C („Zweite verbesserte Ausgabe“ bei Christian Ernst Gabler 1802 in Jena und Leipzig) werden angegeben, sofern sie für die Interpretation von Bedeutung sind. Zitiert wird nach der Gesamtausgabe der Bayerischen Akademie der Wissenschaften unter Angabe der Abteilung, des Bandes und der Seitenzahl.

(GA) Grundlagen der gesammten Wissenschaftslehre als Handschrift für seine Zuhörer 1794/ 95, in: Gesamtausgabe der Bayrischen Akademie der Wissenschaften, Bd. I,2, hg.v. Reinhard Lauth, Hans Jacob, Stuttgart-Bad Cannstatt 1965, S. 249-451.

\section{Werke Hegels}

Die Schriften Hegels werden, soweit verfügbar, nach der Historisch-Kritischen Gesamtausgabe von Meiner zitiert. Bei den „Zusätzen“ der Enzyklopädie der philosophischen Wissenschaften wird auf die zwanzigbändige Werkausgabe des Suhrkamp Verlags zurückgegriffen.

(GW) Gesammelte Werke, in Verbindung mit der Deutschen Forschungsgemeinschaft hg.v. der Nordrhein-Westfälischen Akademie der Wissenschaften und der Künste, Hamburg $1968 \mathrm{ff}$.

(TWA) Werke in zwanzig Bänden, hg.v. Eva Moldenhauer, Karl Markus Michel, Frankfurt am Main 1969-71. 